Subscriber access provided by Caltech Library

Communication

\title{
Visible Wavelength Color Filters using Dielectric Subwavelength Gratings for Backside-illuminated CMOS Image Sensor Technologies
}

Yu Horie, Seunghoon Han, Jeong-Yub Lee, Jaekwan Kim, Yongsung Kim, Amir Arbabi, Changgyun Shin, Lilong Shi, Ehsan Arbabi, Seyedeh Mahsa Kamali, Hong-Seok Lee, Sung Woo Hwang, and Andrei Faraon

Nano Lett., Just Accepted Manuscript • DOI: 10.1021/acs.nanolett.7b00636 • Publication Date (Web): 07 Apr 2017

Downloaded from http://pubs.acs.org on April 10, 2017

\section{Just Accepted}

"Just Accepted" manuscripts have been peer-reviewed and accepted for publication. They are posted online prior to technical editing, formatting for publication and author proofing. The American Chemical Society provides "Just Accepted" as a free service to the research community to expedite the dissemination of scientific material as soon as possible after acceptance. "Just Accepted" manuscripts appear in full in PDF format accompanied by an HTML abstract. "Just Accepted" manuscripts have been fully peer reviewed, but should not be considered the official version of record. They are accessible to all readers and citable by the Digital Object Identifier (DOI®). "Just Accepted" is an optional service offered to authors. Therefore, the "Just Accepted" Web site may not include all articles that will be published in the journal. After a manuscript is technically edited and formatted, it will be removed from the "Just Accepted" Web site and published as an ASAP article. Note that technical editing may introduce minor changes to the manuscript text and/or graphics which could affect content, and all legal disclaimers and ethical guidelines that apply to the journal pertain. ACS cannot be held responsible for errors or consequences arising from the use of information contained in these "Just Accepted" manuscripts. 


\author{
Yu Horie, ${ }^{\dagger, \delta}$ Seunghoon Han, ${ }^{*, \ddagger, \S}$ Jeong-Yub Lee,,$\underset{ }{\ddagger}$ Jaekwan Kim, ${ }^{\ddagger}$ \\ Yongsung Kim,${ }^{\ddagger}$ Amir Arbabi, ${ }^{\dagger}$ Changgyun Shin, ${ }^{\ddagger}$ Lilong Shi, ${ }^{\circledR}$ Ehsan \\ Arbabi, ${ }^{\dagger}$ Seyedeh Mahsa Kamali, ${ }^{\dagger}$ Hong-Seok Lee, ${ }^{\ddagger}$ Sungwoo Hwang,,${ }^{\ddagger}$ \\ and Andrei Faraon $*, \dagger$ \\ $\dagger$ T. J. Watson Laboratory of Applied Physics, California Institute of Technology, 1200 E \\ California Blvd, Pasadena, CA 91125, USA \\ $\ddagger$ Samsung Advanced Institute of Technology, Samsung Electronics, Yeongtong-gu, Suwon-si, \\ Gyeonggi-do, 443-803 South Korea \\ ФAdvanced Image Research Lab, Samsung Electronics, 2 N Lake Ave, Pasadena, CA 91101, USA \\ $\S$ Contributed equally to this work \\ E-mail: shn.han@samsung.com; faraon@caltech.edu
}

\begin{abstract}
We report transmissive color filters based on subwavelength dielectric gratings that can replace conventional dye-based color filters used in backside-illuminated CMOS image sensor (BSI CIS) technologies. The filters are patterned in an 80-nm-thick poly-silicon film on a 115-nm-thick $\mathrm{SiO}_{2}$ spacer layer. They are optimized for operating at the primary RGB colors,


exhibit peak transmittance of $60-80 \%$, and an almost insensitive response over a $\pm 20^{\circ}$ angular range. This technology enables shrinking of the pixel sizes down to near a micrometer.

\section{Keywords}

Subwavelength grating, high-index contrast, color filters, CMOS image sensor

Scalability of complementary metal oxide transistor (CMOS) technology has improved the performance of CMOS image sensors (CIS) in the past decade. One of the trends in the CIS technology, driven mainly by portable devices with small form-factors, is to decrease the pixel size, which will lead to improved spatial resolution for digital imaging. ${ }^{1}$ The scalability of CIS technology has enabled lateral pixel size to be reduced from more than $10 \mu \mathrm{m}$ to less than $2 \mu \mathrm{m}$ in the last decade. $^{2,3}$ Along with scaling of the pixel size, there have been considerable efforts to redesign color filters, ${ }^{4-6}$ microlenses, ${ }^{7}$ and infrared filters ${ }^{8}$ to prevent the degradation of the optical performance. Dye-doped polymers have been conventionally used for RGB color filters in digital color imaging. However, when the pixel size gets smaller, the optical crosstalk among pixels becomes significant because of the small absorption coefficient of the organic dyes. Besides, the dye-doped polymers are essentially photoresists that degrade under ultraviolet illumination or high temperature environments. To address these issues, plasmonic color filters made of metallic thin films with subwavelength patterning have been studied for CIS technology. ${ }^{5,9,10}$ The plasmonic color filters have several advantages such as flexible color tunability across visible spectrum and compatibility with CMOS processes. However, the absolute efficiency of the plasmonic color filters is relatively low (40-50\% range) compared to conventional organic dye-doped filters. Here, we show that dielectric subwavelength gratings can be used to achieve highly efficient transmission color filters with close to angular insensitive properties. Furthermore, compared to dye-based filters, our dielectric-based filters possess better reliability under ultraviolet illumination and at high temperature. For the optimized filter designs, we take the advantage of the backside-illumination (BSI) CIS technologies, where the color filter layer can be placed in close proximity to the photodiodes 
device layer.

Subwavelength gratings provide numerous opportunities to manipulate optical waves especially when the dielectric constant of the grating is high. Owing to the subwavelength size and high-index contrast, the subwavelength structures can be engineered to support relatively strong optical resonances that can enhance the spectral features. ${ }^{11}$ Various designs have been studied for various applications, including visible wavelengths structural colors, ${ }^{12-14}$ RGB color filters for polarization-sensitive transmission filter ${ }^{15}$ and polarization-insensitive reflective RGB color filters, ${ }^{16}$ anti-reflection coating, ${ }^{17,18}$ and reflective-transmission filters for displays. ${ }^{19}$ In this letter, we demonstrate designs for polarization insensitive filters made of polycrystalline silicon (poly$\mathrm{Si}$ ) located on $\mathrm{SiO}_{2}$ spacer layer that separates them from the Si photodiodes layer (Figure 1(a)). For ease of scalable fabrication, we also restrict the thicknesses to be equal among the set of red $(\mathrm{R})$, green $(\mathrm{G})$ and blue (B) filters. Our material choice for the gratings is poly-Si that has a high refractive index $(n>3.5)$ as well as sufficiently low absorption $(\kappa<0.2)$ over the entire visible wavelength range (see Supporting Information).

We optimized the shape parameters for the primary set of RGB transmissive color filters based on the similarity to our reference filter spectra and luminance signal-to-noise ratio (YSNR) in color image sensors. ${ }^{20}$ The optimum structure is an 80 -nm-thick poly-Si slab with air holes, placed on a 115-nm-thick $\mathrm{SiO}_{2}$ spacer layer sitting on BSI Si photodiodes. The red design has a period of $250 \mathrm{~nm}$ with a $90 \mathrm{~nm}$ hole diameter in hexagonal lattice, the green design has a period of $180 \mathrm{~nm}$ with a $140 \mathrm{~nm}$ hole diameter in a square lattice, and the blue design has a period of $270 \mathrm{~nm}$ with a $240 \mathrm{~nm}$ hole diameter in a hexagonal lattice. The transmittance spectra of the filters are computed by rigorous coupled wave analysis (RCWA) technique, shown in Figure 1(b). The simulations include the effect of the $\mathrm{SiO}_{2}$ spacer layer. The interference between the light passing through the high-index-contrast grating layer and the reflection from the underlying Si photodiodes layer, results in a color-selective transmission spectrum with peak transmittance of $60-80 \%$. Based on calculations, substantial YSNRs are expected by calculation, when applying the IR-cut filter spectrum of the lens modules and the image signal processing pipeline. ${ }^{21}$ For average color error 4 , 
YSNR $=28.4$ at low light condition (20 lux) and YSNR $=39.9$ at high light condition (700 lux) were obtained. We note that using current color filters technology the YSNR values are smaller: YSNR $=22.0$ at 20 lux and YSNR $=33.3$ at 700 lux. The chromaticity coordinates of the optimized color filters when a conventional IR-cut filter is applied are shown in CIE 1931 chromaticity diagram (Figure 1(c)). Because of the rotational symmetry of the structures, the spectral response is polarization-insensitive for normal incidence. Also, importantly, the filters exhibit relatively insensitive angular responses for each color as can be seen in Figure 1(d), which is crucial for maintaining the transmitted color when shrinking the pixel size, as further discussed in the letter.

The optimized color filters were fabricated using poly-Si materials prepared by amorphous-Si deposition followed by a high-temperature furnace annealing method ${ }^{22-25}$ (see Sample fabrication and Supporting Information). In our proof-of-concept experiments, instead of directly measuring transmission through the designed filter structures into the photodiode Si layers, we characterized the reflection from the subwavelength grating with a $\mathrm{SiO}_{2}$ spacer layer fabricated on a Si substrate, and also the transmission through the subwavelength grating layer with the same design fabricated on a transparent quartz substrate. Scanning electron microscope (SEM) images of the fabricated color filters are shown in Figure 2(a). To characterize the reflection/transmission spectra for the fabricated color filters, we used a custom built confocal microscope setup, in which only the spectrum in the field of view was coupled to a visible spectrometer. From the magnification of the relay lens, we effectively collected the reflection/transmission spectrum from $6 \mu \mathrm{m}$ circular apertures (see Measurement procedure for details). In Figure 2(b,c), reflection and transmission spectra (solid line) are shown along with the RCWA-simulated spectra (dotted line). Both reflection and transmission spectra for each color filter design show good agreement between their simulation and measurement. Note that the reason that the transmission and reflection are not complementary is because the reflectance graphs in Figure 2(b) account for the interference between the light passing through the high-index-contrast grating layer and the reflection from the underlying $\mathrm{Si}$ photodiodes layer.

To investigate the effect of the finite size pixels on the filtering properties, we characterized 
the transmission microscope color images for the samples fabricated on the quartz substrate. We fabricated several pixels with sizes of $30 \mu \mathrm{m}, 5 \mu \mathrm{m}, 3 \mu \mathrm{m}$, and $1 \mu \mathrm{m}$. The corresponding optical microscope images are shown in Figure 3(a,b) and the SEM image of the $1 \mu \mathrm{m}$-pixel Bayer pattern is shown in Figure 3(c). For the red and green color filters, the transmitted colors of the images appear to be preserved even up to the pixel size of $1 \mu \mathrm{m}$ (the number of holes is $5 \times 4$ hole array for red and $6 \times 6$ hole array for green, Figure 3(c)). For the blue color filter, the transmitted color appears to be preserved up to $3 \mu \mathrm{m}$ pixel size, but exhibits a different color for the pixel size of $1 \mu \mathrm{m}$ (the number of holes is $4 \times 3$ hole array, Figure 3(c)). To understand the change in the imaged color for different filter designs, we simulated the transmission spectra for the finite size of pixels using the plane wave expansion (PWE) method. From the viewpoint of the diffraction theory, transmission through a finite sized pixel is equivalent to transmission of a set of plane waves incident from different angles through an infinite pixel. The angular distribution of the plane waves are dictated by the size and shape of the finite pixel and the amplitude of each plane wave is modified by the transmission coefficient of the infinite pixel. Therefore, the smaller the pixel size, the broader the angular distribution of plane waves. In other words, to preserve the color spectrum when the pixel size is $w$, the transmission coefficient of the periodic arrays should remain constant up to the maximum angle given by $\sin ^{-1}(\lambda / w)$, the first zero of the spatial frequency spectrum of a square aperture, where $\lambda$ is the wavelegth of the light. To verify this, we measured the transmission spectra for the set of color filters fabricated on the quartz substrate under off-axis illuminations for $0^{\circ}, 10^{\circ}, 20^{\circ}$, and $30^{\circ}$. The plots are shown in Figure 3(d). The measured spectra show good agreement with the RCWA-simulated spectra (also see Figure S3 in Supporting Information). As is expected from the results of the color imaging results for the fabricated finite size pixels, the red and green filters are less angularly sensitive than the blue filter. From Figure 1(d) (also see Figure S1 and Figure S2 in Supporting Information), we can see that the blue filter can be considered angularly insensitive up to $\sim 20^{\circ}$, indicating the acceptable pixel size for the presented blue design is $w=\lambda /(\sin \theta) \sim 1.4 \mu \mathrm{m}$ (for $\lambda=475 \mathrm{~nm}$ ), which agrees well with the optical microscope color imaging results. Since the measured filters are composed of the subwavelength 
grating sitting on a quartz substrate, the measured angular response for the transmission discussed so far does not include the interference effect between the light passing through the subwavelength grating layer and the reflection from the underlying Si photodiodes layer. However, the effect of such interference on the angular response is not significant as analyzed in Figure S2 in Supporting Information. Therefore, the actual angular performance as well as the effect of small pixel size for the designed filter should behave similarly to the measured filters.

In conclusion, we demonstrated transmissive color filters based on subwavelength dielectric gratings, optimized for the primary RGB colors for BSI CIS technologies. The optimized set of color filters exhibits efficient peak transmittance of $60-80 \%$, superior to plasmonic color filters, owing to the combination of high refractive index contrast and relatively low loss in poly-Si. We also studied the effect of finite size pixels, comparable to the pixel size available in state-of-the-art CIS technologies, in conjunction with the angular response of the infinite array of the structures and found that the designed filters preserve their colors down to near a micrometer. We note that the proposed dielectric grating color-filters are highly customizable and compatible with micrometer and sub-micrometer pixel sizes, when considering new image processing methods such as an RWB (Red, White, Blue) matrix. ${ }^{21}$ To make the filter fabrication more compatible with the CMOS backend processes, a pulsed laser annealing process ${ }^{26}$ may be used for preparing low-loss poly-Si layers as low temperature alternative methods, instead of the high temperature annealing method. Furthermore, by exploiting the freedom of designing spatially varying phase, amplitude, and polarization of the transmission ${ }^{27-32}$ that subwavelength gratings are capable of, not only the conventional dye-doped color filters but also the microlenses on top of CIS pixels could be replaced in one layer of dielectric subwavelength grating on top of Si photodiodes layers. 


\section{Methods}

\section{Optical simulation.}

To optimize the transmission spectra of color filters, the RCWA technique was used to simulate the periodic array of subwavelengths structures. The experimentally measured complex refractive index data for poly-Si was used for the entire visible spectra. The complex refractive index for poly-Si is shown in Figure S4 in Supporting Information.

\section{Sample fabrication.}

Amorphous-silicon thin film was deposited by low-pressure chemical vapor deposition (LPCVD) method on a Si substrate as well as on a quartz substrate. Then the films were crystallized to polySi of low extinction coefficient using furnace annealing at $950^{\circ} \mathrm{C}$. Nano-hole patterns of ZEP520A resist were defined on the poly-Si surface using electron beam lithography methods. The ZEP520A resist served as a mask for dry etching of the 80-nm-thick silicon layer. $\mathrm{A}_{4} \mathrm{~F}_{8}$ and $\mathrm{SF}_{6}$ mixture gas was used for dry etching of poly-Si and forming pattern profiles with right angle. Residual resist was removed by sulfuric acid.

\section{Measurement procedure.}

For reflection/transmission spectral measurements, we used a custom built confocal microscope setup illustrated in Figure S5 in Supporting Information. We used a fiber-coupled collimated light from a Tungsten-Halogen lamp (HL-2000-HP, Ocean Optics) as broad-band light source. The sample was illuminated with the collimated light and imaged by a pair of $20 \times$ infinity-corrected objective lens (LMPLFLN20x, Olympus) and a tube lens with a focal length of $15 \mathrm{~mm}$ onto a pinhole with diameter of $100 \mu \mathrm{m}$ to select the region of interest. The spatially filtered light was being coupled into a spectrometer (SpectraPro-2500i, Princeton Instruments). The sample was mounted on a rotation mount to measure the transmission spectra for different incident angles. Backside- 
illuminated optical microscope images were taken by Axio Observer inverted microscope (Carl Zeiss) equipped with a digital color CCD camera (Infinity 2-3, Lumenera Corp.). The sample was illuminated from the backside of the quartz substrate with a halogen lamp.

\section{Supporting Information Available}

Angular dependence of the color filters for $s$ - and $p$-polarized light, angular dependence of the color filters with and without interference effect of the $\mathrm{SiO}_{2}$ spacer layer, measured transmission spectra as well as simulation results for $s$ - and $p$-polarized light, complex refractive index data measured for poly-Si, and schematic of the measurement setup used. This material is available free of charge via the Internet at http://pubs.acs.org/.

\section{Acknowledgement}

This work was supported by Samsung Electronics. Part of the device nanofabrication was developed at the Kavli Nanoscience Institute at California Institute of Technology. Y. H. was supported by a Japan Student Services Organization (JASSO) fellowship. S. M. K. was supported by the DOE Light-Material Interactions in Energy Conversion Energy Frontier Research Center funded by the US Department of Energy, Office of Science, Office of Basic Energy Sciences under Award no. DE-SC0001293. We thank H. A. Atwater and B. Fultz for providing the transmission-type microscope and the spectrometer.

\section{Author Information}

\section{Corresponding Authors}

* E-mails:

A.F.: faraon@caltech.edu

S.H.: shn.han@samsung.com 


\author{
Author Contributions \\ $\S$ Equal author contribution. \\ Notes \\ The authors declare no competing financial interest.
}

\title{
References
}

(1) Ahn, J.; Moon, C.-R.; Kim, B.; Lee, K.; Kim, Y.; Lim, M.; Lee, W.; Park, H.; Moon, K.; Yoo, J.; Lee, Y. Y. Y.; Park, B.; Jung, S.; Lee, J.; Lee, T.-H.; Lee, Y. Y. Y.; Jung, J.; Kim, J.H.; Kim, T.-C.; Cho, H.; Lee, D.; Lee, Y. Y. Y. Advanced image sensor technology for pixel scaling down toward $1.0 \mu \mathrm{m}$. 2008 IEEE International Electron Devices Meeting. 2008; pp $1-4$.

(2) Catrysse, P. B.; Wandell, B. A. Proc. SPIE 2005, 5678, 1-13.

(3) Kriss, M. Handbook of Digital Imaging, 1st ed.; Wiley, 2015; pp 31-84.

(4) Yu, Y.; Wen, L.; Song, S.; Chen, Q. J. Nanomater. 2014, 2014, 1-17.

(5) Yokogawa, S.; Burgos, S. P.; Atwater, H. A. Nano Lett. 2012, 12, 4349-4354.

(6) Park, H.; Dan, Y.; Seo, K.; Yu, Y. J.; Duane, P. K.; Wober, M.; Crozier, K. B. Nano Lett. 2014, 14, 1804-1809.

(7) Huo, Y.; Fesenmaier, C. C.; Catrysse, P. B. Opt. Express 2010, 18, 5861-5872.

(8) Frey, L.; Parrein, P.; Raby, J.; Pellé, C.; Hérault, D.; Marty, M.; Michailos, J. Opt. Express 2011, 19, 13073-13080.

(9) Chen, Q.; Chitnis, D.; Walls, K.; Drysdale, T. D.; Collins, S.; Cumming, D. R. S. IEEE Photonics Technol. Lett. 2012, 24, 197-199. 
(10) Burgos, S. P.; Yokogawa, S.; Atwater, H. A. ACS Nano 2013, 7, 10038-10047.

(11) Brongersma, M. L.; Cui, Y.; Fan, S. Nat. Mater. 2014, 13, 451-460.

(12) Cao, L.; Fan, P.; Barnard, E. S.; Brown, A. M.; Brongersma, M. L. Nano Lett. 2010, 10, 2649-2654.

(13) Proust, J.; Bedu, F.; Gallas, B.; Ozerov, I.; Bonod, N. ACS Nano 2016, 10, 7761-7767.

(14) Vashistha, V.; Vaidya, G.; Serebryannikov, A. E.; Bonod, N.; Krawczyk, M. arXiv.org, e-Print Arch., Phys. 2016, 1-9.

(15) Kanamori, Y.; Shimono, M.; Hane, K. IEEE Photonics Technol. Lett. 2006, 18, 2126-2128.

(16) Kanamori, Y.; Ozaki, T.; Hane, K. Opt. Express 2014, 22, 25663-25672.

(17) Kanamori, Y.; Sasaki, M.; Hane, K. Opt. Lett. 1999, 24, 1422-1424.

(18) Proust, J.; Fehrembach, A.-L.; Bedu, F.; Ozerov, I.; Bonod, N. Sci. Rep. 2016, 6, 24947.

(19) Regan, E. C.; Shen, Y.; Massuda, A.; Miller, O. D.; Soljačić, M. arXiv.org, e-Print Arch., Phys. 2016,

(20) Alakarhu, J. Image sensors and image quality in mobile phones. Proceedings of International Image Sensor Workshop. 2007.

(21) Mlinar, M.; Keelan, B. Imaging systems with clear filter pixels. Patent US 20130242148, 2013.

(22) Ruther, R.; Livingstone, J.; Dytlewski, N. Thin Solid Films 1997, 310, 67-74.

(23) Buitrago, R. H.; Risso, G. A.; Cutrera, M.; Battioni, M.; Bernardez, L. D.; Schmidt, J. A.; Arce, R. D.; Koropecki, R. R. Int. J. Hydrogen Energy 2008, 33, 3522-3525.

(24) Preston, K.; Schmidt, B.; Lipson, M. Opt. Express 2007, 15, 17283-17290. 
(25) Zhu, S.; Fang, Q.; Yu, M. B.; Lo, G. Q.; Kwong, D. L. Opt. Express 2009, 17, 20891-20899.

(26) Lee, J.; Song, B.; Kim, J.; Lee, C.-W.; Han, S.; Baik, C.-W.; Jeong, H.; Kim, Y.; Lee, C. S. Proc. SPIE 2016, 9927, 992708-992708-11.

(27) Fattal, D.; Li, J.; Peng, Z.; Fiorentino, M.; Beausoleil, R. G. Nat. Photonics 2010, 4, 466-470.

(28) Lu, F.; Sedgwick, F. G.; Karagodsky, V.; Chase, C.; Chang-Hasnain, C. J. Opt. Express 2010, $18,12606-14$.

(29) Arbabi, A.; Horie, Y.; Ball, A. J.; Bagheri, M.; Faraon, A. Nat. Commun. 2015, 6, 1-10.

(30) Arbabi, A.; Horie, Y.; Bagheri, M.; Faraon, A. Nat. Nanotechnol. 2015, 10, 937-943.

(31) Jahani, S.; Jacob, Z. Nat. Nanotechnol. 2016, 11, 23-36.

(32) Genevet, P.; Capasso, F.; Aieta, F.; Khorasaninejad, M.; Devlin, R. Optica 2017, 4, 139-152. 
(a)

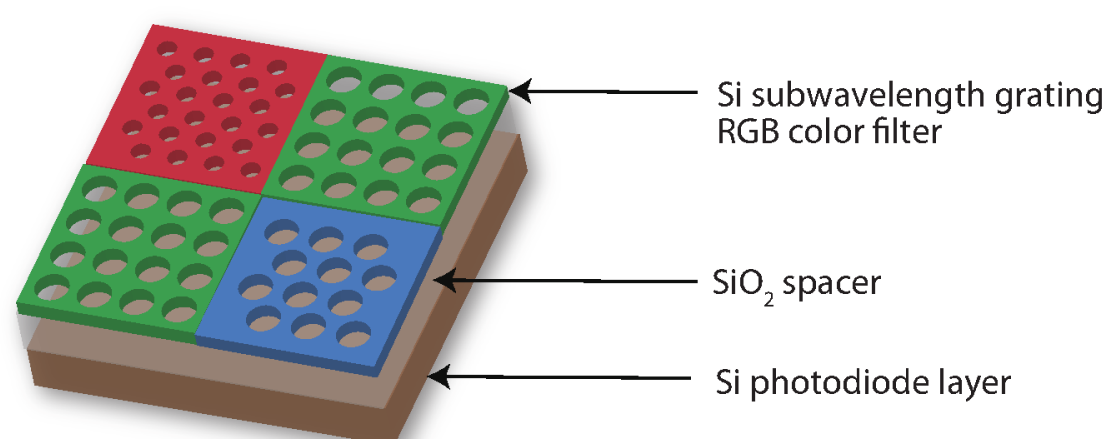

(b)

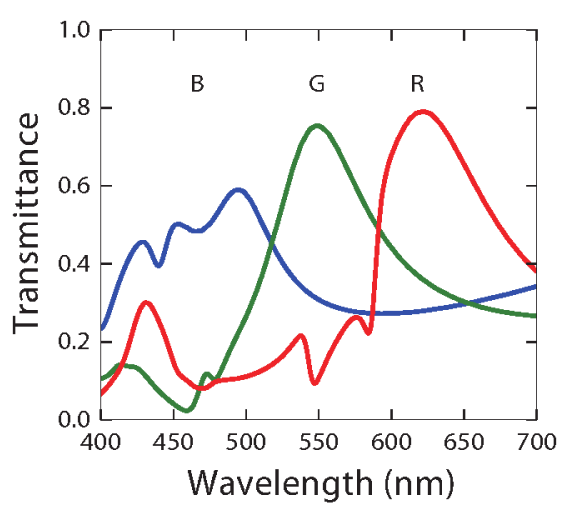

(d)

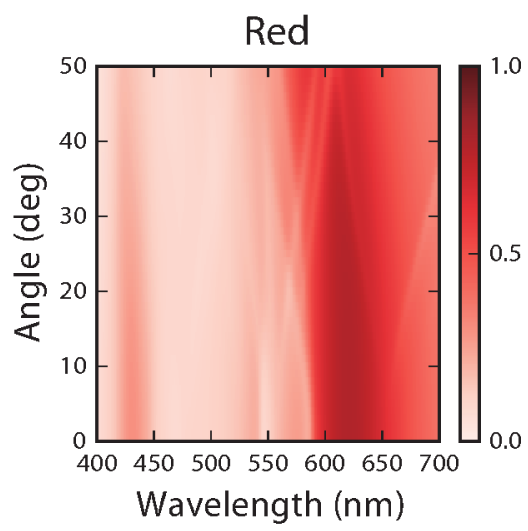

(c)

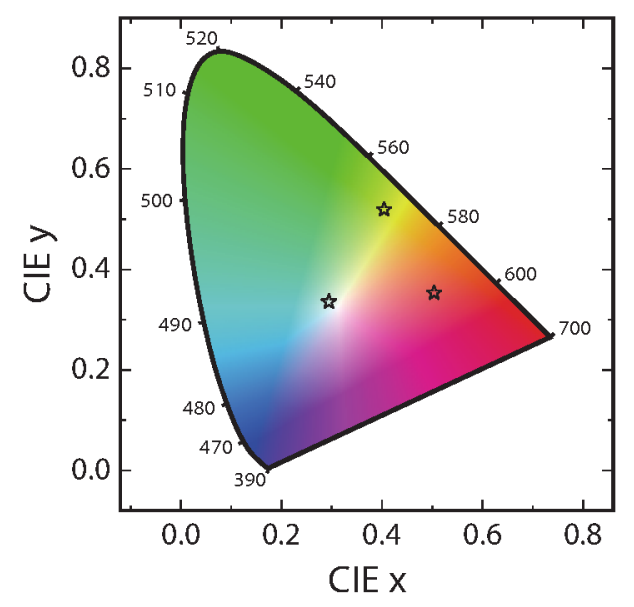

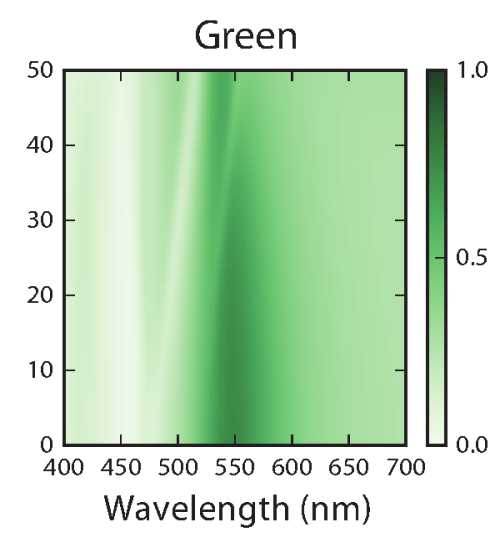

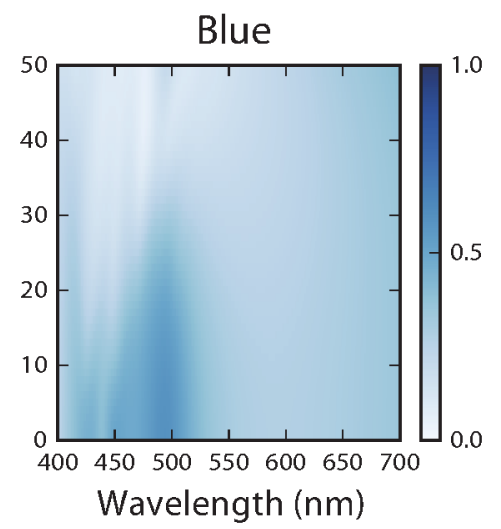

Figure 1: Visible wavelength on-chip color filters using dielectric subwavelength gratings for BSICIS applications. (a) Schematic illustration of the realization of the Bayer pattern using dielectric subwavelength grating color filters. The design consists of a patterned 80-nm-thick poly-Si slab on a 115-nm-thick $\mathrm{SiO}_{2}$ spacer layer sitting on BSI Si photodiodes. (b) RCWA simulation results of the transmission spectra under normal incidence, optimized for the RGB primary colors. (c) Chromaticity coordinates of the optimized color filters in CIE 1931 chromaticity diagram. (d) RCWA simulations of the angular dependence of the corresponding transmission spectra for unpolarized light (See Figure S1 in Supporting Information for polarization dependence). 
(a)
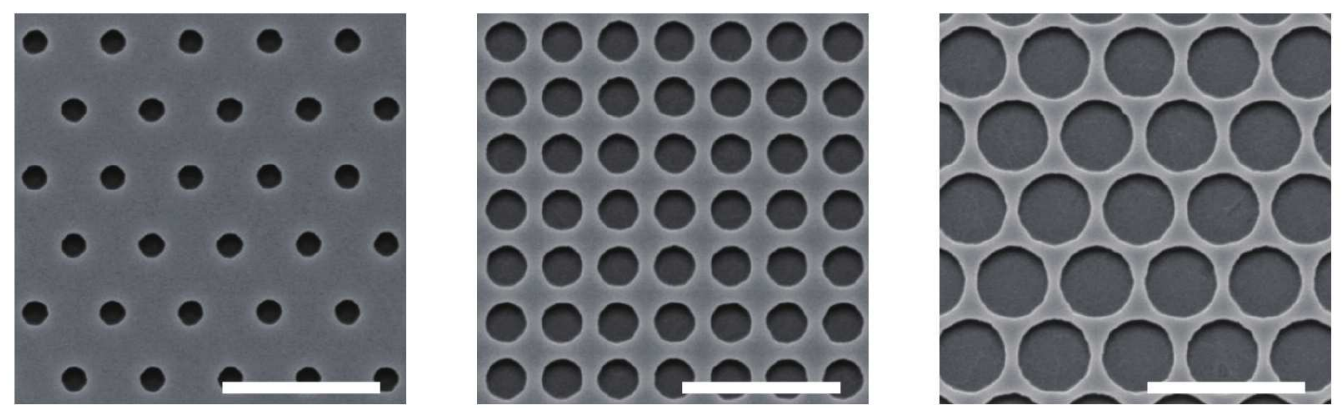

(b)
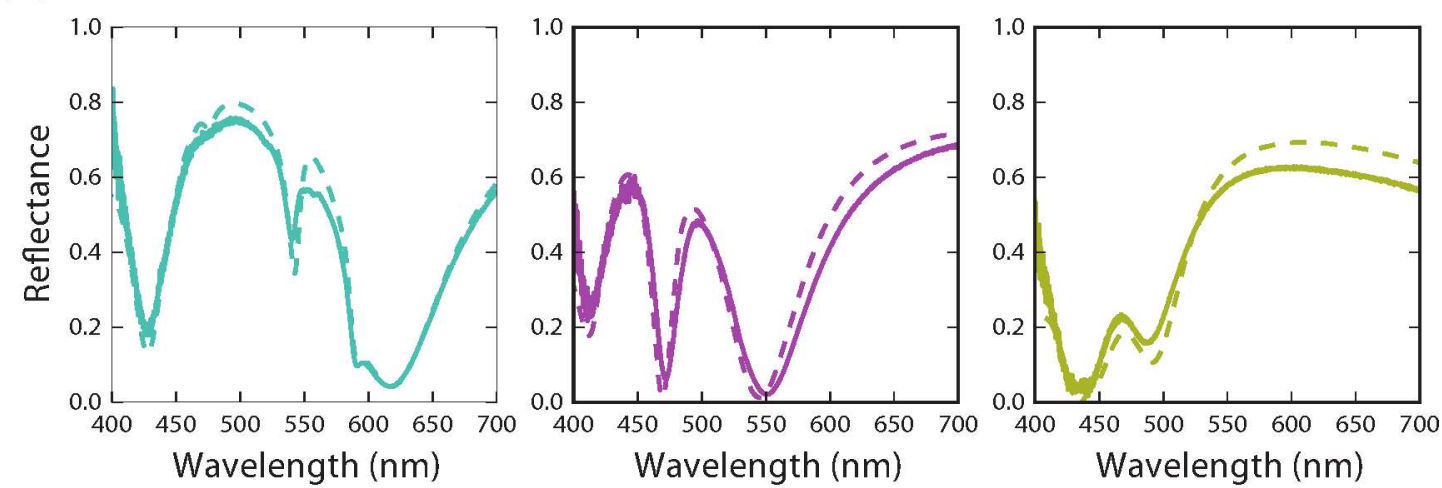

(c)
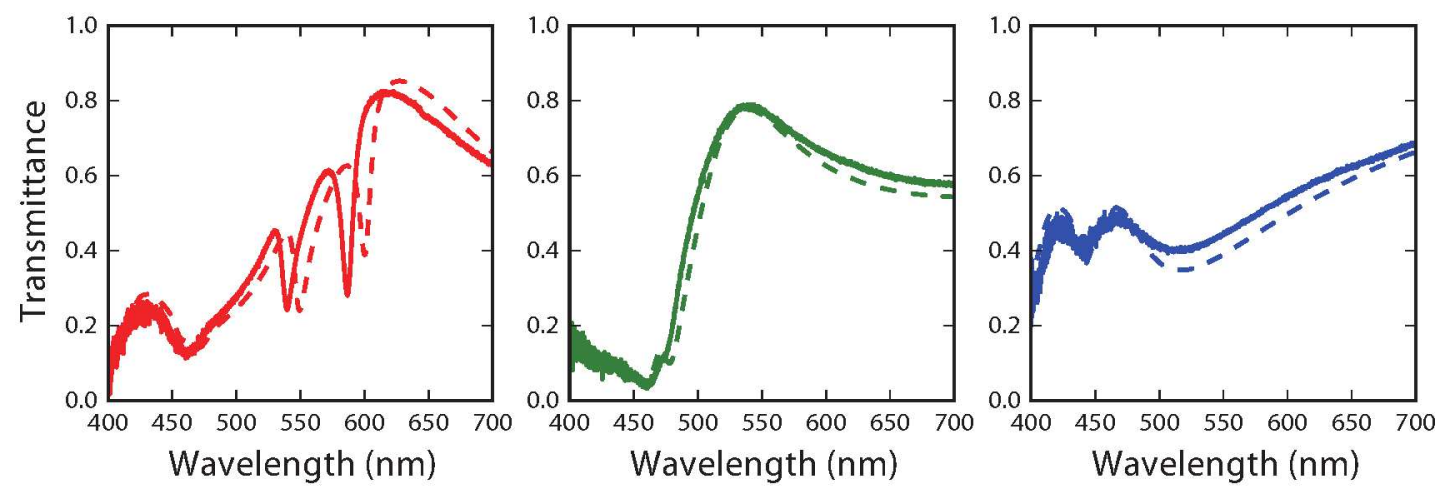

Figure 2: Fabrication and measurement results of visible color filters on Si substrates with $115 \mathrm{~nm}$ thick $\mathrm{SiO}_{2}$ spacer layer or on quartz substrates. (a) Scanning electron microscope (SEM) images of the fabricated color filters. The scale bars are $500 \mathrm{~nm}$. (b) Measured reflection spectra for the designed color filters on $\mathrm{Si}$ substrates with $115 \mathrm{~nm}$-thick $\mathrm{SiO}_{2}$ spacer layer. The corresponding simulated reflection spectra are also shown in dotted lines. (c) Measured transmission spectra for the designed color filters on quartz substrates. The corresponding simulated transmission spectra are also shown in dotted lines. Note that the reason that the transmission and reflection are not complementary is because the results shown in (b) are affected by the interference effect between the light passing through the high-index-contrast grating layer and the reflection from the underlying Si photodiodes layer, while such interference effect does not happen in devices shown in (c). 
(a)

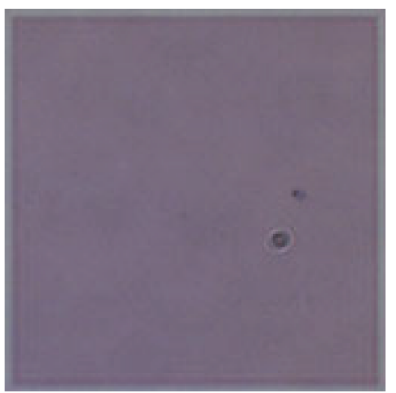

(b)

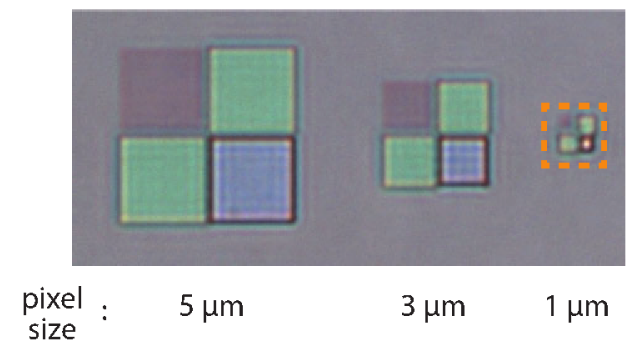

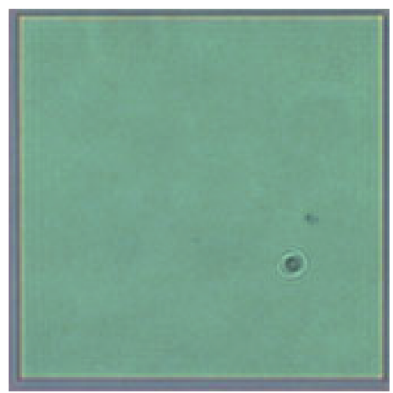

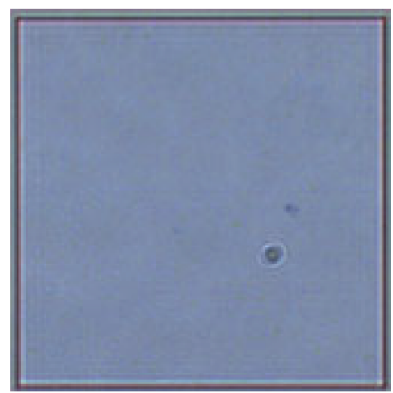

(c)

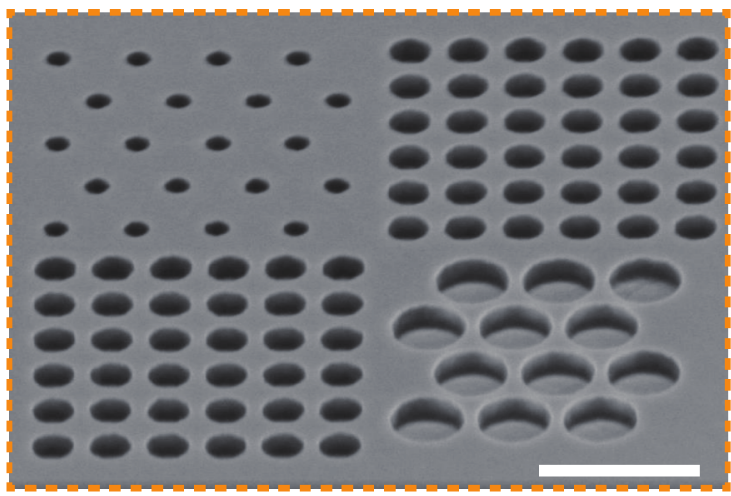

(d)
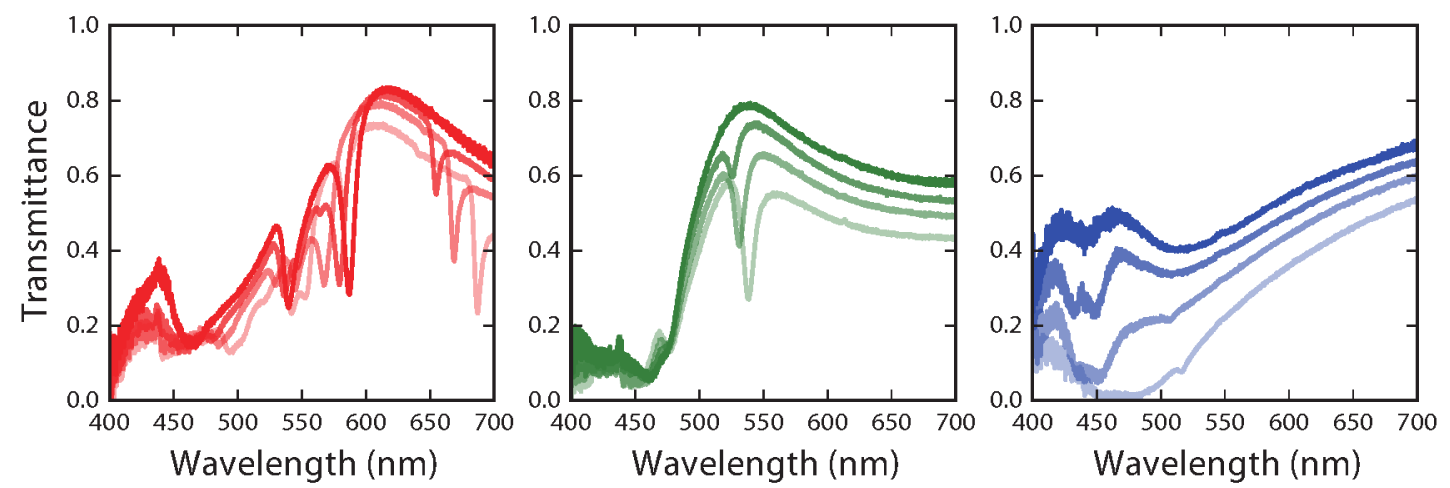

Figure 3: Spectral responses for small pixel sizes. (a) Backside-illuminated optical microscope images for the fabricated RGB color filters with large pixel size $(30 \mu \mathrm{m})$ on quartz substrates. (b) The Bayer pattern demonstrations of small pixel size designs $(5 \mu \mathrm{m}, 3 \mu \mathrm{m}$, and $1 \mu \mathrm{m})$. The transmitted colors are preserved even with smaller pixel sizes. (c) The SEM image of the $1 \mu \mathrm{m}$-pixel color filters in the Bayer pattern, corresponding to the smallest Bayer pattern optical microscope image in (b). The scale bar is $500 \mathrm{~nm}$. (d) Measured transmission spectra of each color filter with $s$-polarized oblique illuminations. For each filter, transmission spectra for incidence angles of $0^{\circ}, 10^{\circ}, 20^{\circ}$, and $30^{\circ}$ are shown with successively lighter shades (i.e. darkest is $0^{\circ}$, and lightest is $30^{\circ}$ ). Simulated spectra as well as $p$-polarized cases are shown in Figure $\mathbf{S 3}$ in Supporting Information. 


\section{Graphical TOC Entry}

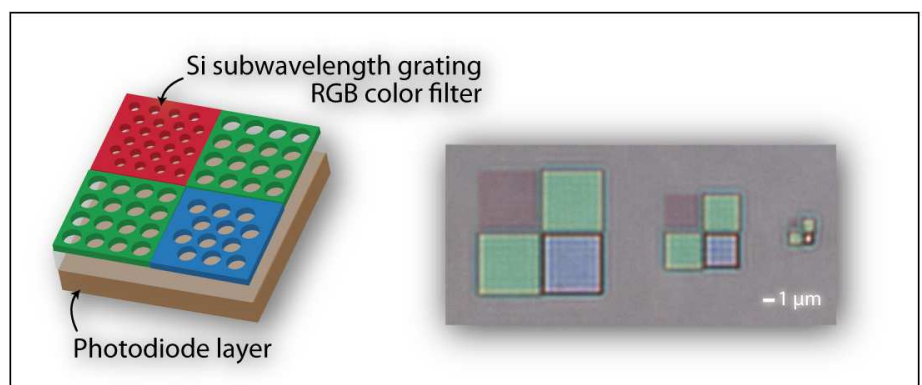

Micro-scale realization of the RGB Bayer patterns using Si dielectric subwavelength grating color filters 
(a)
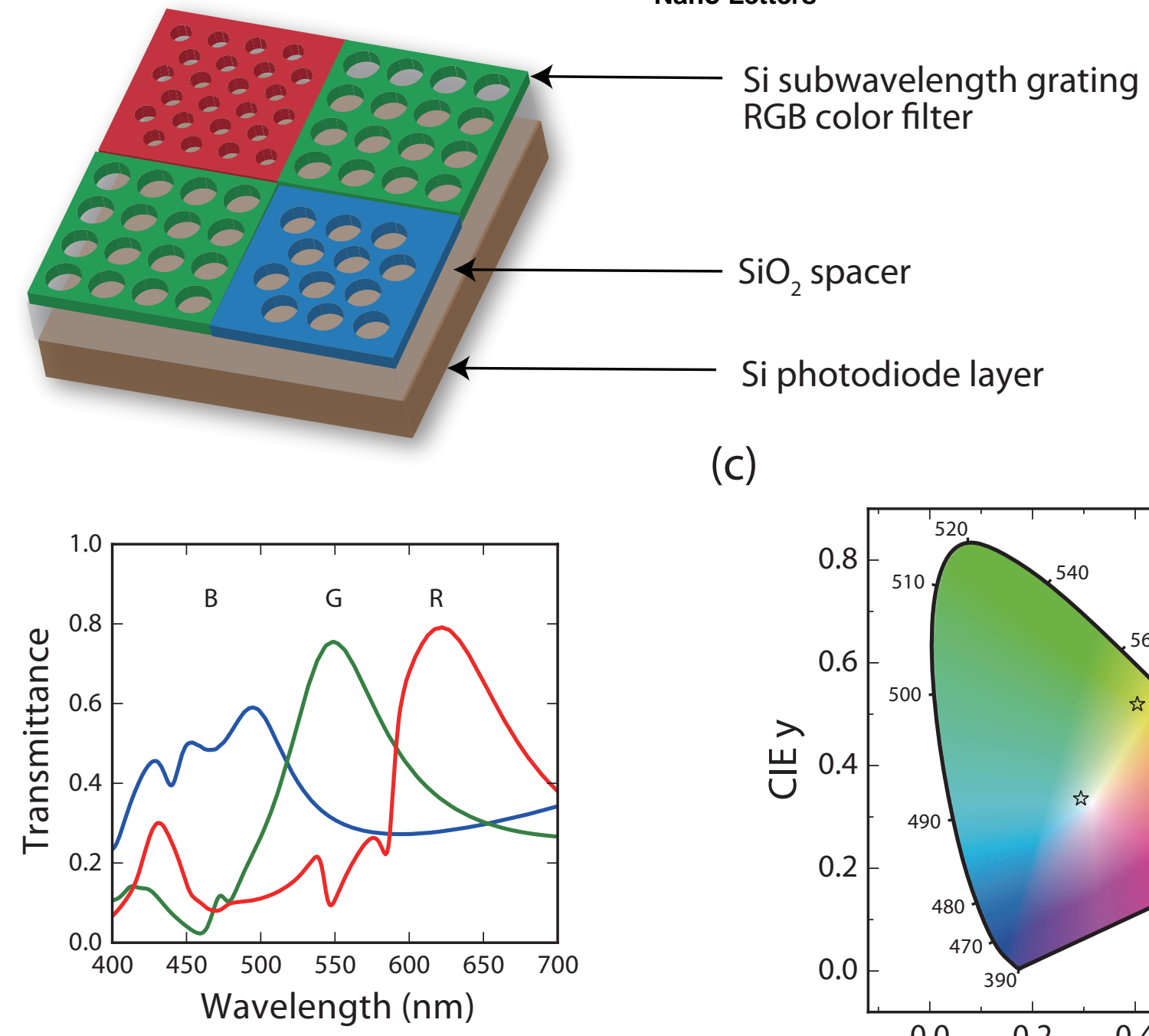

$\mathrm{SiO}_{2}$ spacer

Si photodiode layer

(c)

Red
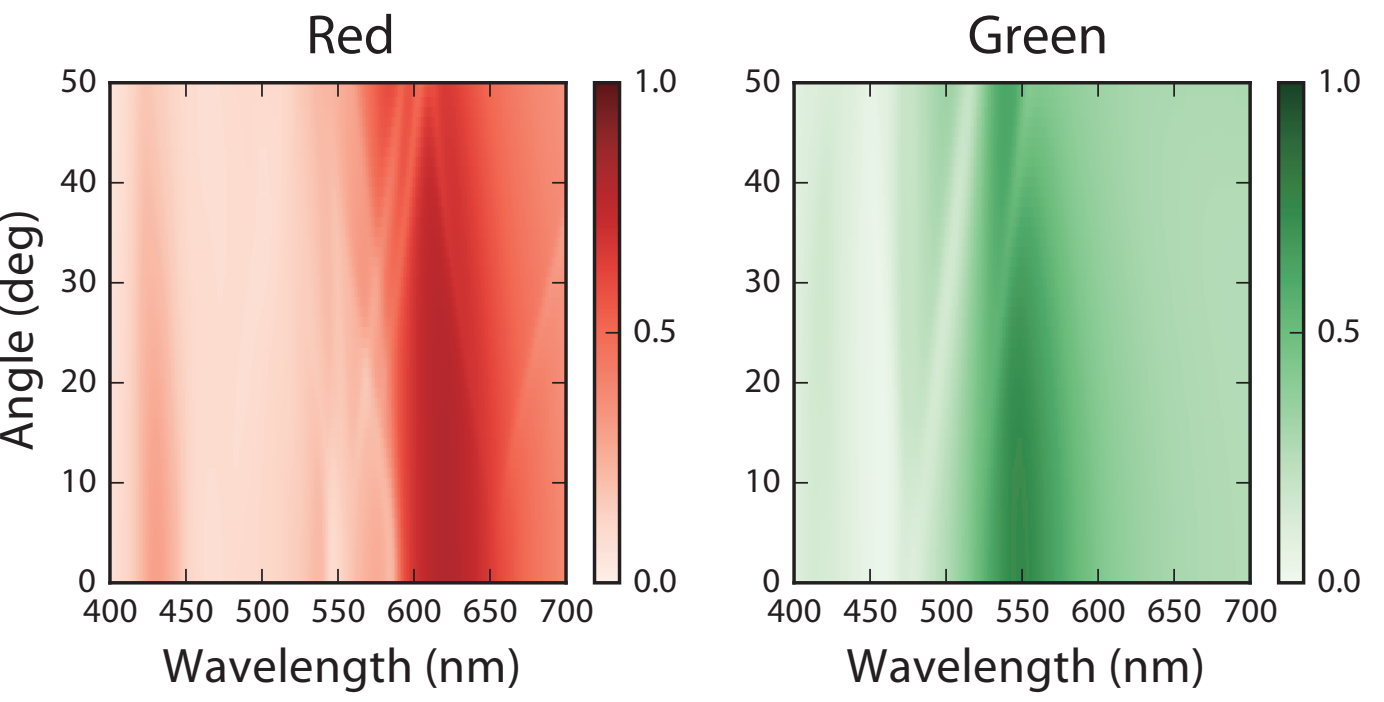

CIE x
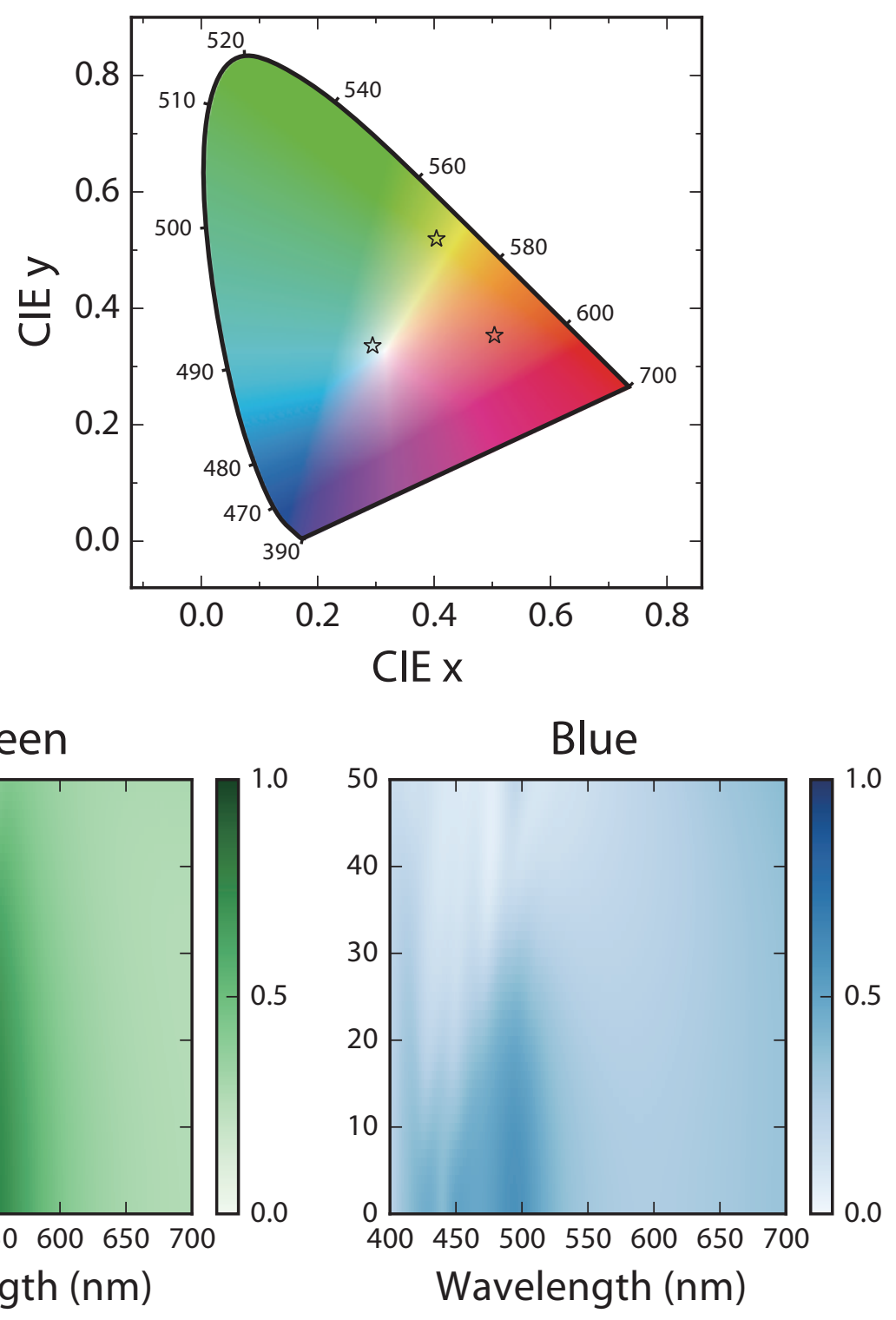

ACS Paragon Plus Environment 


\section{Page 17 of 18}

\section{1}

2

3

4

5

6

7

8

9

10

11

12

$1(\mathrm{~b})$

14

15

16

17

18

19

20

21

22

23 व

24

25

26

27

28

29

30

$3(\mathrm{C})$

32

33

34

35

36

37

38

39

40

41

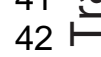

43

44

45

46

47

\section{Nano Letters}
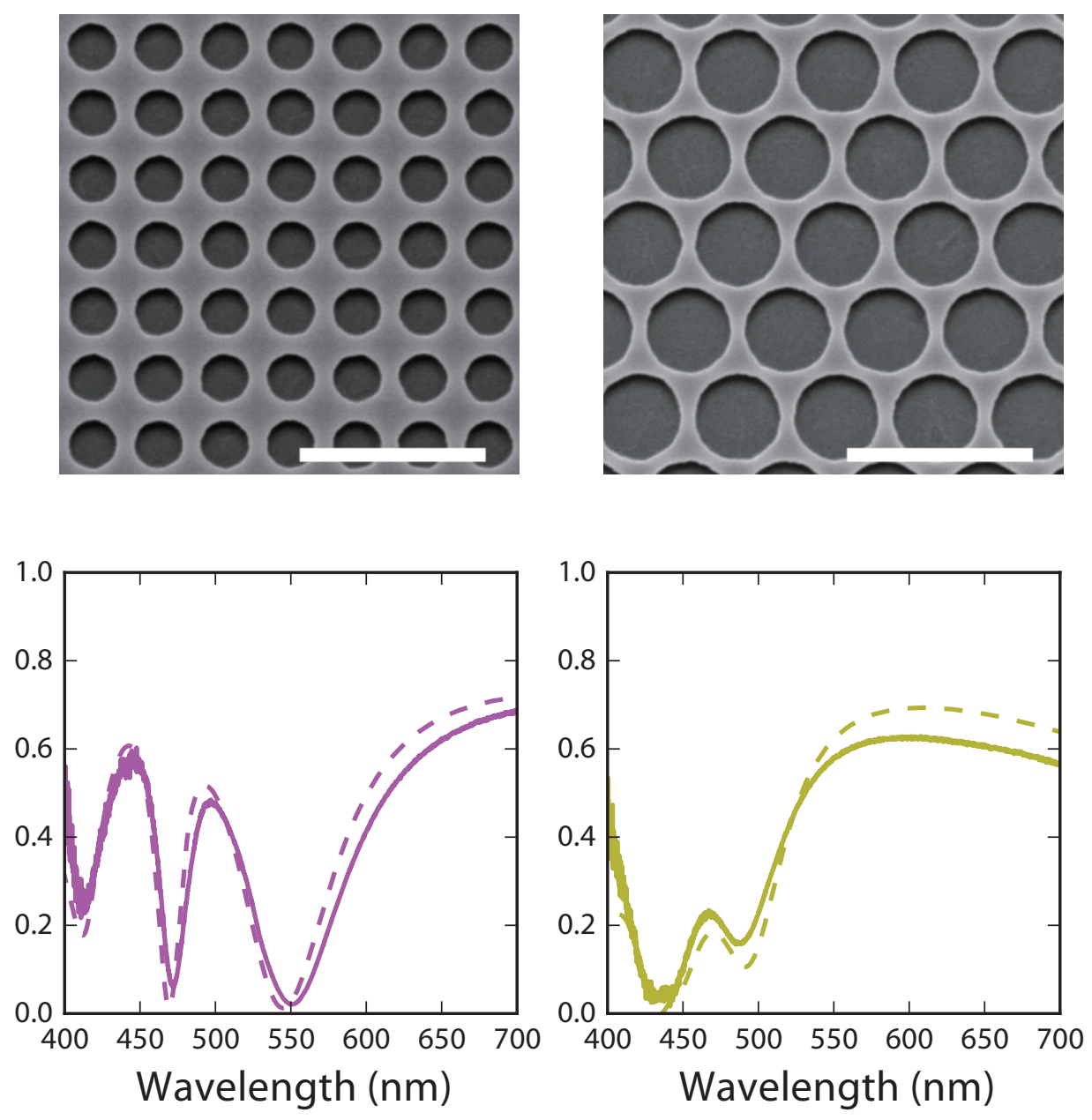
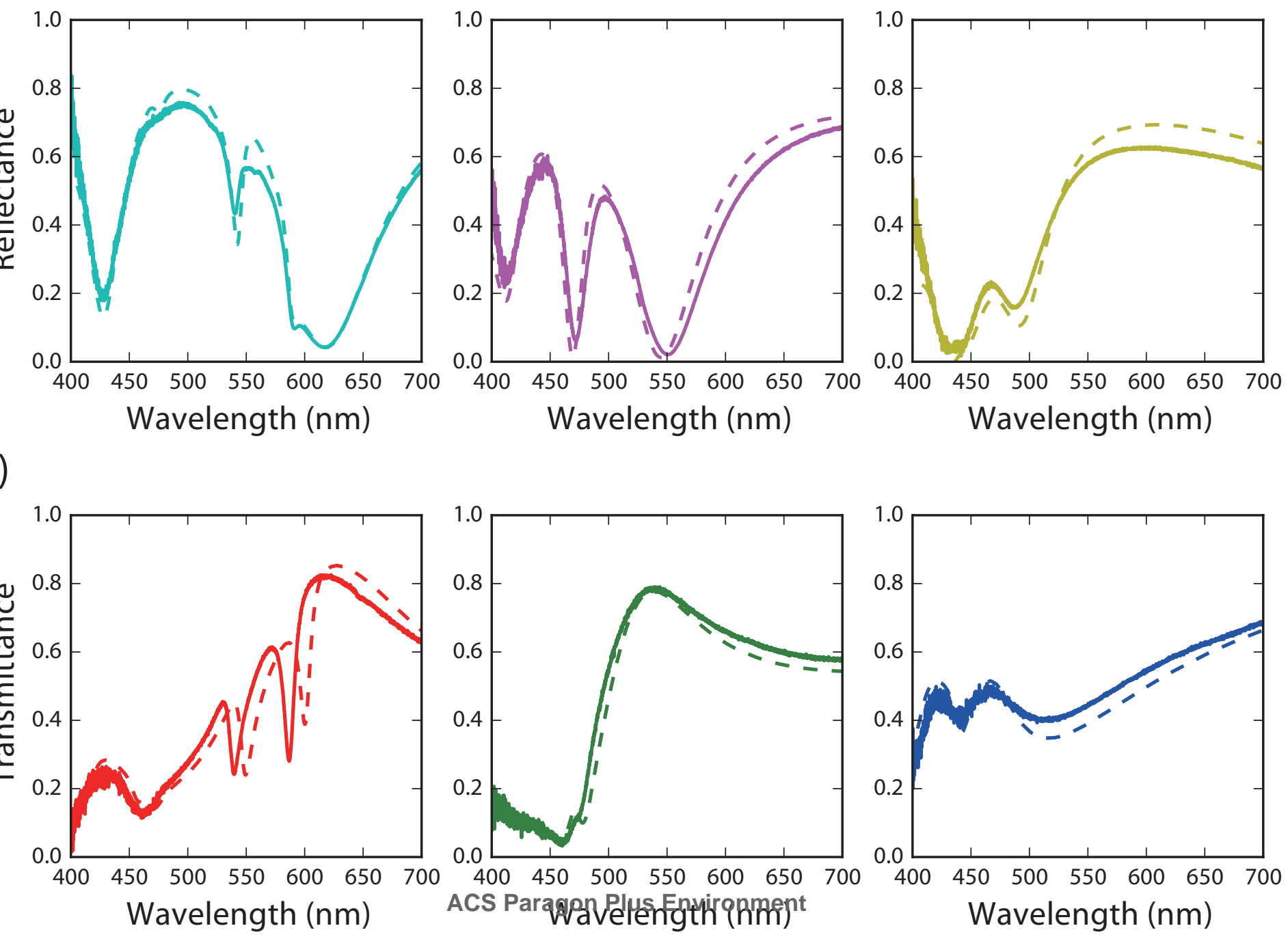
(a)

1
2
3
4
5
6
7
8
9
10
11
12

$13(b)$

15

16

17

18

19

20

21

22

23

24

25

2

2

28

29

30

3

$3 \Varangle d)$

33

34

35

36

37

3

38

39

40

41

41

42

43

45

46

47

48

49

\section{Nano Letters}

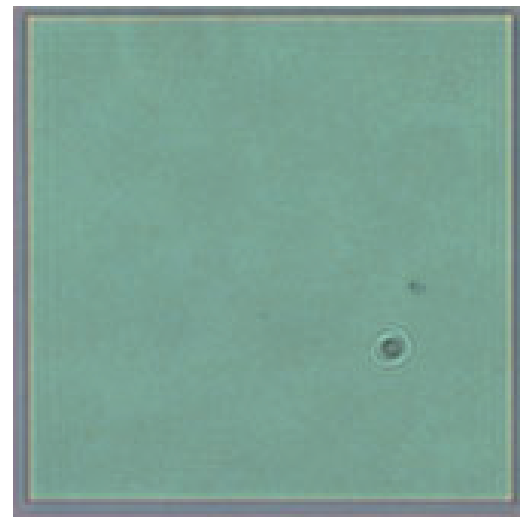

(c)
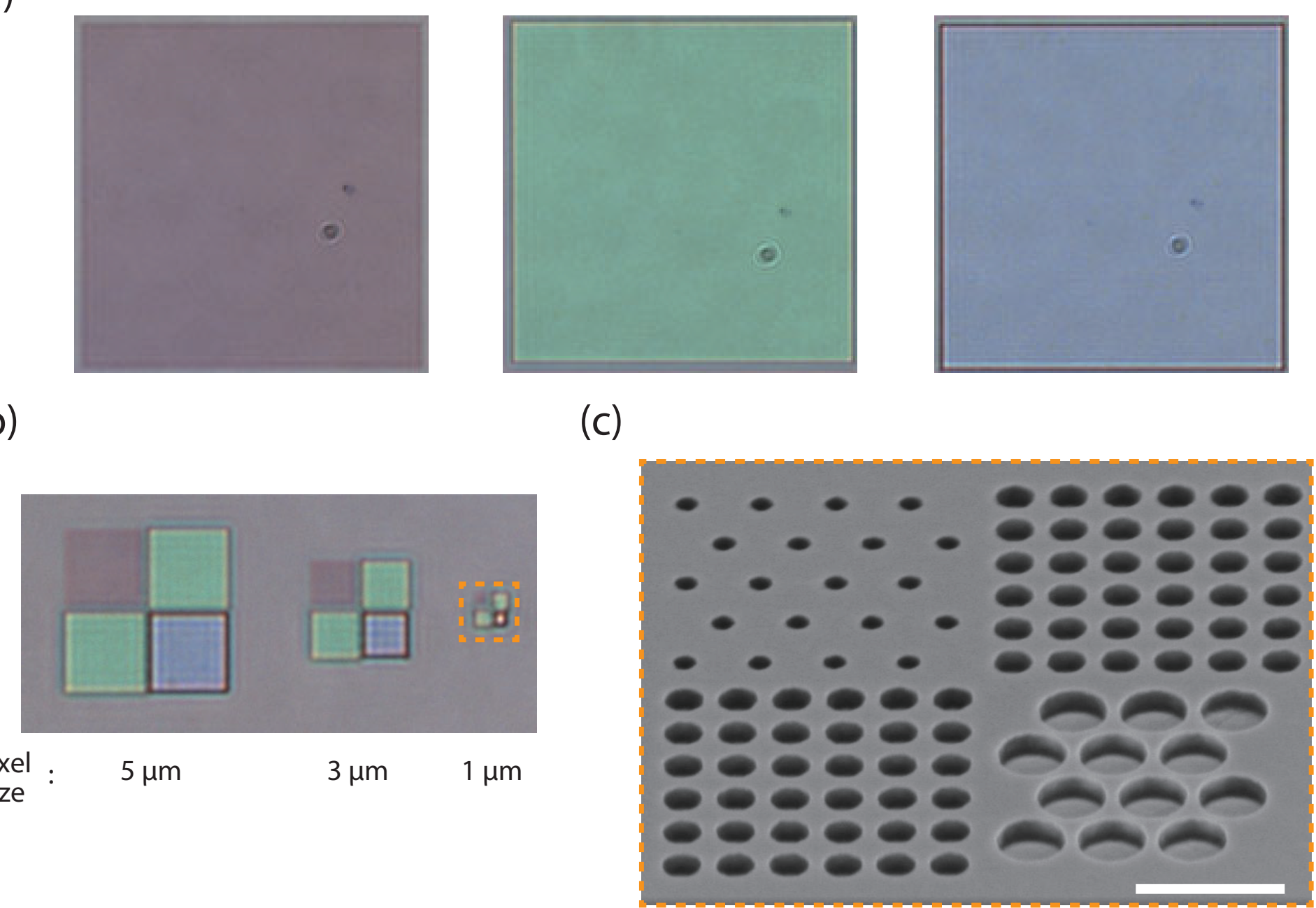
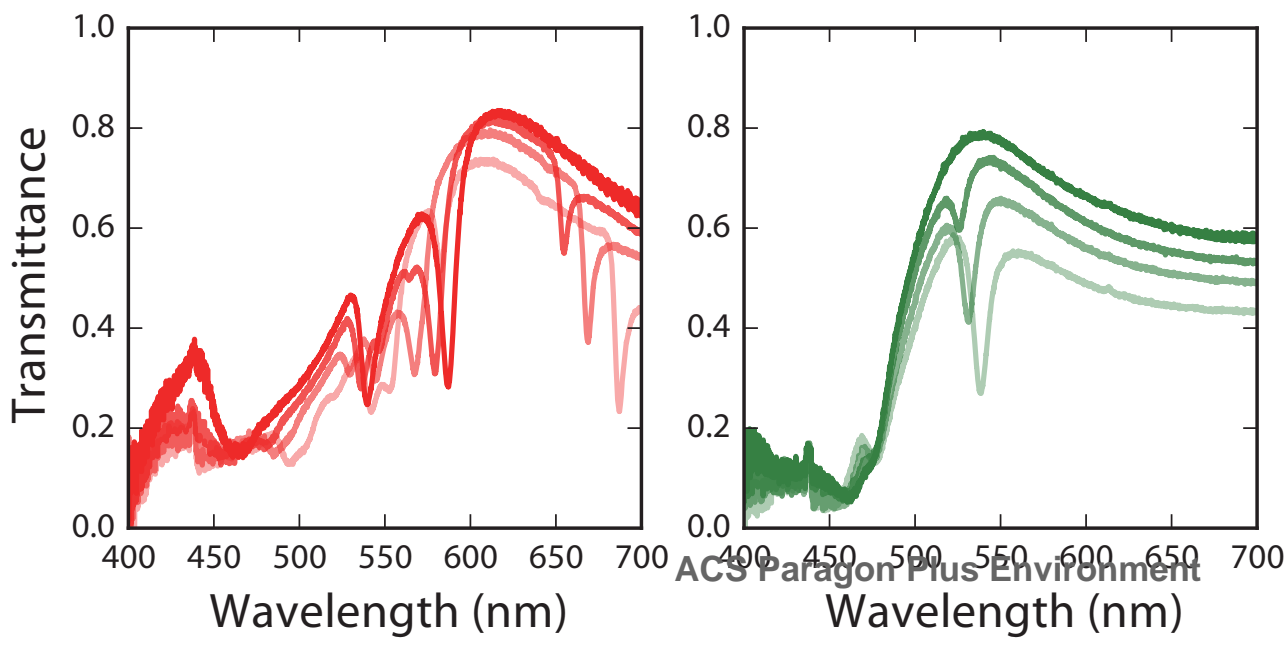

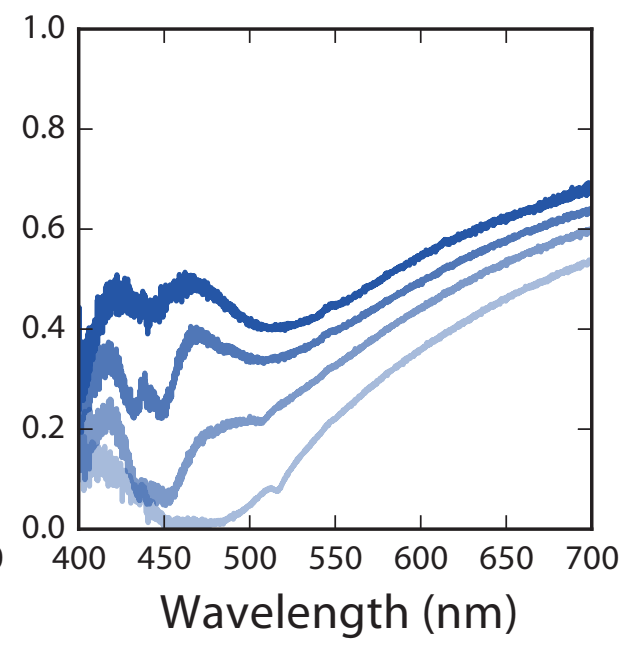

